\title{
The early evolution of tidal dwarf galaxies
}

\author{
S. Recchi ${ }^{1,2}$, C. Theis ${ }^{1}$, P. Kroupa ${ }^{3}$, and G. Hensler ${ }^{1}$ \\ 1 Institute of Astronomy, Vienna University, Türkenschanzstrasse 17, 1180 Vienna, Austria \\ e-mail: [recchi; theis; hensler] @astro.univie.ac.at \\ 2 INAF - Osservatorio Astronomico di Trieste, via G.B. Tiepolo 11, 34131 Trieste, Italy \\ e-mail: recchi@oats.inaf.it \\ 3 Argelander Institute for Astronomy, Bonn University, Auf dem Hügel 71, 53121 Bonn, Germany \\ e-mail: pavel@astro.uni-bonn.de
}

Received 9 February 2007 / Accepted 29 May 2007

\section{ABSTRACT}

\begin{abstract}
Context. Dwarf galaxies can arise from self-gravitating structures emerging from tidal tails. What fraction of the known dwarf galaxies in the Local Universe can have this origin is still a matter of debate.

Aims. In our effort to understand the origin and evolution of tidal dwarf galaxies and their correspondence with local objects, the first step is to understand how these galaxies (which are supposed to have a limited amount of dark matter) react to the feedback of the ongoing star formation.

Methods. We make use of 2-D chemodynamical calculations in order to study the early evolution of isolated, dark matter-free dwarf galaxies. We present models in which feedback parameters are varied. We also compare the results with dark matter-dominated dwarf galaxy models.

Results. All the considered models show that the star formation proceeds for more than $300 \mathrm{Myr}$, therefore dwarf galaxies without large dark matter halos are not necessarily quickly destroyed. The chemical evolution of these objects is consistent with the main chemical properties of the dSphs of the Local Group. Models with large dark matter halos show results consistent with models free of dark matter, indicating that the distribution of gas is more important than the depth of the potential well in determining the global behaviour of dSph-sized dwarf galaxies.
\end{abstract}

Key words. ISM: abundances - ISM: bubbles - ISM: jets and outflows - galaxies: evolution

\section{Introduction}

Galactic collisions and close encounters are thought to be common events in the Local Universe. It has been clear for some time that the structure of a galaxy can be affected by tidal interactions with close neighbours (Toomre \& Toomre 1972). Even for what concerns the Milky Way, signs of the interaction with the Magellanic Clouds have been analysed in detail (e.g. Mastropietro et al. 2005; Ružička et al. 2007).

There is growing evidence that tidal arms, produced by the interaction of gas-rich galaxies, can trigger the formation of selfgravitating structures evolving into objects similar to the local dwarf galaxies (Duc \& Mirabel 1998; Walter et al. 2006). These objects are therefore called tidal dwarf galaxies (TDGs) and their production rate must have been higher in the early universe than today, due to the higher probability of tidal interactions and the larger gas content of young galaxies. From a stellar dynamical point of view, it is shown that these star complexes typically survive the first few hundred Myr (Kroupa 1998) and can evolve into objects similar to the present-day dwarf spheroidal (dSph) galaxies (Kroupa 1997; Metz \& Kroupa 2007). Recently, detailed numerical simulations of the evolution of tidal tails have been presented (Bournaud \& Duc 2006; Wetzstein et al. 2007) showing that the formation of TDGs in these sites is favoured by the presence of large and extended reservoirs of gas. These studies also show that TDGs must have a very reduced dark matter (DM) content, confirming previous results (e.g. Barnes $\&$ Hernquist 1992). Okazaki \& Taniguchi (2000) even point out that the majority, if not all the dwarf galaxies could be TDGs if only a few long-lived TDGs emanate from each late-type encounter.

It is therefore important to understand the early evolution of TDGs, in order to clarify how the potentially disruptive feedback from the ongoing star formation (SF) acts on a DM-poor structure, characterised by a very reduced potential well, and to compare them with DM-rich models. Finally, the study of the chemical evolution of dwarf galaxies and comparison with observed ones offers a wealth of information about the past history and the origin of these objects.

In this paper we study for the first time the 2-D chemodynamical evolution of DM-free model galaxies, in order to understand under which conditions these objects can sustain the energy released by the dying stars without experiencing a complete blow-away, as well as what the final chemical patterns are. This work is an extension of the 1-D chemodynamical simulations of DM-free dwarf galaxies of Hensler et al. (2004). In Sect. 2 we describe the adopted model and in Sect. 3 we summarise the main results of our investigations. Finally, in Sect. 4 we draw some conclusions.

\section{The model}

We performed 2-D simulations of the dynamical and chemical evolution of TDGs in cylindrical coordinates. The hydro solver and the implementation of the routines for the chemical 
evolution have been described in Recchi et al. (2001, 2004) and references therein. Several new implementations have been added in this version and will be comprehensively described in Recchi et al. (in preparation). The main code extensions are:

- self-gravity like in Rieschick \& Hensler (2003), which solves the Poisson equation by means of the so-called "Alternate Direction Implicit" method;

- metal-dependent stellar wind luminosities taken from the Starburst99 (Leitherer et al. 1999) software package;

- a star formation rate proportional, within each grid cell, to the amount of gas (through a constant $\varepsilon_{\mathrm{SF}}$ ) with an upper temperature threshold $\left(T_{\mathrm{thr}}\right)$ and valid only for convergent gas flows (i.e. when $\nabla \cdot \boldsymbol{v}<0$ ). $\varepsilon_{\mathrm{SF}}$ denotes the fraction of gas converted to stars within the next $5 \mathrm{Myr}$, once the SF criteria are fulfilled.

The calculation strategy is that: the SF criteria identify the grid points that, within an interval of $5 \mathrm{Myr}$, produce stars. The star masses are distributed according to a Salpeter IMF. All the relevant variables concerning this SF generation are stored in arrays that are used to calculate the feedback from SNeIa, SNeII, and intermediate-mass stars. In this way, the chemical composition (initial $Z=0$ ) of stars is always recorded and metal-dependent yields and energy production can be taken into consideration properly. In spite of some tricks to save memory, the calculations are very computationally demanding (several weeks to months for each run), therefore only a limited number of runs can be performed. For our convenience, the dynamics of the stars is neglected in this set of models, therefore the stars remain where they are born. Due to their short lifetimes, this is not critical at all for the massive stars, which are the main sources of stellar energy feedback.

Although these simulations are not aimed at reproducing specific objects, the Local Group dwarf galaxy Fornax gives us a good reference for calibrating the initial setup. Indeed, it has been suggested that the dSphs near to the Milky Way may be TDGs because their spatial distribution is incompatible with a cosmological origin (Kroupa et al. 2005). Although Local Group $\mathrm{dSphs}$ are commonly believed to be DM-dominated, there are arguments suggesting that they are not necessarily DM-rich. Twisted and squashed isophotes are common in Local Group $\mathrm{dSphs}$ and are not compatible with the presence of a massive DM halo. Most of the kinematical properties of these objects can also be explained without DM (Metz \& Kroupa 2007). An extensive discussion about this point will be presented in Recchi et al. (in preparation). The initial (metal-free) gas distribution is spherically symmetric and follows a King profile with a core radius of $500 \mathrm{pc}$. The total gas mass in the grid (a sphere of $\sim 8 \mathrm{kpc}$ of radius) is calculated according to $M_{\mathrm{g}}=M_{\text {Fornax }} / \varepsilon_{\mathrm{SF}}, M_{\text {Fornax }}$ being the mass of Fornax (assumed to be $6.8 \times 10^{7} M_{\odot}$, Mateo 1998).

We consider models in which the SF efficiency $\varepsilon_{\mathrm{SF}}$ and the temperature threshold $T_{\text {thr }}$ are varied. Two models with a large rigid DM halo are considered, too, in order to outline the differences with DM-free models. The central DM density is calculated according to the observed velocity dispersion of Fornax (10.5 $\mathrm{km} \mathrm{s}^{-1}$, Mateo 1998) and the distribution follows a quasiisothermal profile with core radii of $1 \mathrm{kpc}$ and $200 \mathrm{pc}$, respectively. These models contain $1.1 \times 10^{7}$ and $3.7 \times 10^{7} M_{\odot}$ of DM within $1 \mathrm{kpc}$, respectively. We also consider a model in which the feedback from stellar winds is suppressed. Model parameters are summarised in Table 1. A wider range of values of $\varepsilon_{\mathrm{SF}}$ and $T_{\text {thr }}$ have been considered, as well as models with better spatial resolution and models with an initial anisotropic distribution
Table 1. Model parameters.

\begin{tabular}{ccccc}
\hline \hline Model & DM? (core radius) & $\varepsilon_{\mathrm{SF}}$ & $T_{\text {thr }}(\mathrm{K})$ & Stellar winds? \\
\hline 1 & No & 0.1 & $10^{4}$ & Yes \\
$1 \mathrm{dc}$ & Yes $(200 \mathrm{pc})$ & 0.1 & $10^{4}$ & Yes \\
$1 \mathrm{de}$ & Yes $(1 \mathrm{kpc})$ & 0.1 & $10^{4}$ & Yes \\
2 & No & 0.1 & $5 \times 10^{3}$ & Yes \\
3 & No & 0.1 & $10^{4}$ & No \\
4 & No & 0.05 & $10^{4}$ & Yes \\
\hline
\end{tabular}
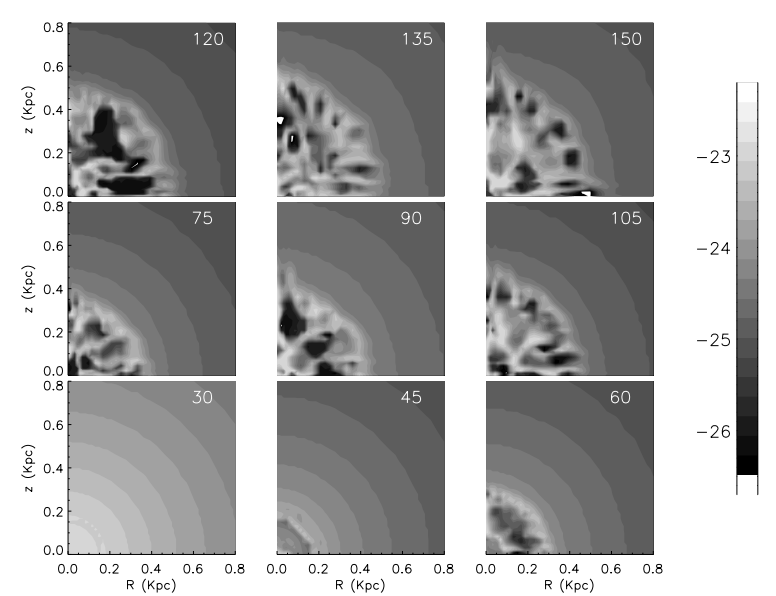

Fig. 1. Density contours of the gas for Model 1 at 9 evolutionary times (labelled, in Myr, at the top right corner of each panel). The density scale $\left(\right.$ in $\mathrm{g} \mathrm{cm}^{-3}$ ) is on the righthand strip.

of gas. For the sake of simplicity, we focus in this letter only on the 6 basic models, mentioning briefly the behaviour of the other models. Only the first 300 Myr of the evolution have been calculated. This is due to the large computational time required by the simulations, but also to the fact that for timescales longer than this, interaction effects with surrounding galaxies are supposed to play a major role in the evolution of these small objects. The effect of tidal interactions on the evolution of these objects is currently under investigation.

\section{Results}

\subsection{A typical evolutionary sequence}

We show here an example of the chemodynamical evolution of an isolated, DM-free dwarf galaxy. This model (designated as Model 1, see Table 1) is characterised by $\varepsilon_{\mathrm{SF}}=0.1$ and $T_{\mathrm{thr}}=10^{4} \mathrm{~K}$. This temperature is much higher than the typical temperatures of the cores of molecular clouds, where SF takes place. Indeed the size of each computational cell is also much larger than a molecular cloud core. (The size of the first cell is $10 \mathrm{pc}$, then it proceeds radially outwards with a size ratio between adjacent zones of 1.028.) Since this temperature has to be considered as the average temperature of a region of the galaxy encompassing a star-forming molecular cloud, $T_{\text {thr }}$ is necessarily a free parameter in our models. We discuss its effect in Sect. 3.2.

The evolution of Model 1 during the first $150 \mathrm{Myr}$ is shown in Fig. 1. During the first few $10^{7} \mathrm{yr}$, the central density is not high enough to trigger SF; therefore, the system is only characterised by a slow infall motion, due to the self-gravity. After $\sim 30 \mathrm{Myr}$ the gas has been condensed enough to form the first stars. A network of cavities with increasing size and complexity is created due to the patchy distribution of the SF sites. It 


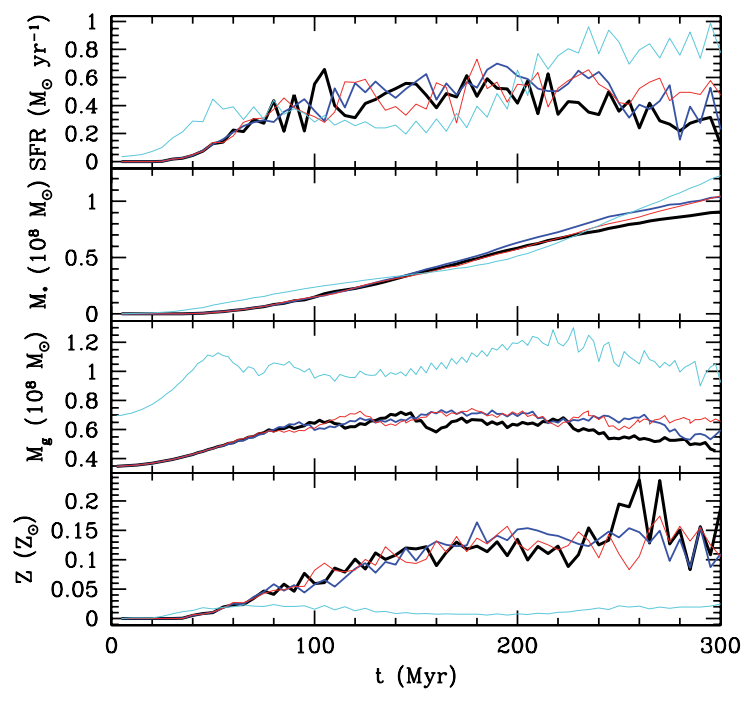

Fig. 2. Evolution with time of the SFR (in $M_{\odot} / \mathrm{yr}$, upper panel), stellar mass (in $10^{8} M_{\odot}$, second panel), gas mass within $1 \mathrm{kpc}$ (in $10^{8} M_{\odot}$, third panel), and mean metallicity of the stellar component (in $Z_{\odot}$, lower panel) for Model 1 (reference model, very thick lines), Model 2 (reduced $T_{\text {thr }}$, thick lines), Model 3 (no stellar winds, thin lines), and Model 4 (reduced $\varepsilon_{\mathrm{SF}}$, very thin lines).

maintains, however, a spherical symmetry and creates a gradually expanding supershell. The superbubble grows slowly in size, reaching $\sim 600 \mathrm{pc}$ in $150 \mathrm{Myr}$. Only at $t \sim 200 \mathrm{Myr}$ does the size of the superbubble reach $1 \mathrm{kpc}$, and at this point the SF rate (SFR) begins to decrease, due to the reduction of available gas. At the end of the simulation, it is approximately one fourth of its peak value (see Fig. 2). The evolution of gas mass with time in the central kpc of Model 1 (Fig. 2, third panel) is very similar to the evolution of the SFR with time. This is not a surprise, since we have chosen a linear dependence between SF and the gas mass. The increase in the gas mass in the first $\sim 150 \mathrm{Myr}$ is due to the fact that the density distribution extends up to several $\mathrm{kpc}$ and the self-gravity attracts a fraction of the gas located in the external regions. The global metallicity of the stellar component (Fig. 2, lower panel) oscillates around a mean value of $\sim 0.1 Z_{\odot}$, consistent with what is observed in Fornax and in the largest dSphs of the Local Group (Mateo 1998). For this model we checked the timescale required to blow-away the gas from the central kpc, and this corresponds to $\sim 400 \mathrm{Myr}$. We reran this model with higher spatial resolution, i.e. a resolution of $5 \mathrm{pc}$. This model shows a very similar behaviour to Model 1 .

With our model, we are also able to check the build-up of the $[\mathrm{O} / \mathrm{Fe}]$ vs. $[\mathrm{Fe} / \mathrm{H}]$ relation in the stellar population. This is shown in Fig. 3. The evolution in the first few $10^{7} \mathrm{yr}$ is dominated by SNeII ejecta and this produces a plateau of $[\mathrm{O} / \mathrm{Fe}]$. At $[\mathrm{Fe} / \mathrm{H}] \sim-2$, the Fe produced by the SNeIa (which explode with some delay compared to SNeII, Matteucci \& Greggio 1986) starts reducing the $[\mathrm{O} / \mathrm{Fe}]$. For Local Group dSphs, a knee at $[\mathrm{Fe} / \mathrm{H}] \sim-2$ (much earlier than the analogous knee observed in Milky Way stars) is a common feature (see e.g. Tolstoy et al. 2003; Lanfranchi \& Matteucci 2004).

\subsection{A parametric study}

With its lower temperature threshold for the onset of SF, Model 2 is supposed to be characterised by a reduced impact of the feedback and therefore a milder SFR due to the fact that a smaller amount of gas fulfils the SF condition. Indeed, its behaviour

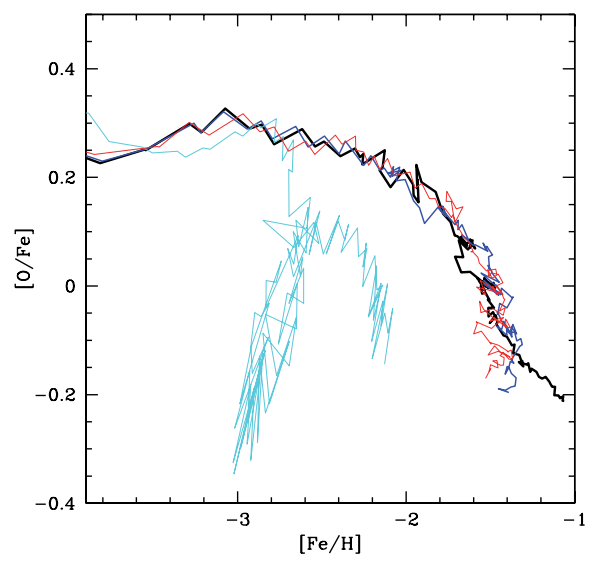

Fig. 3. $[\mathrm{O} / \mathrm{Fe}]$ vs. $[\mathrm{Fe} / \mathrm{H}]$ in stars. Symbols as in Fig. 2.

(Figs. 2 and 3) is similar to the reference model, showing that SF self-regulation (Köppen et al. 1995) is at work, similar to that in 1 -D chemodynamical modelling of dwarf galaxies (Hensler et al. 2004).

In Model 3 we have suppressed the input of energy coming from stellar winds, thereby reducing the feedback. There is a significant effect on the global thermal energy budget, resulting in a slightly larger amount of gas inside the galaxy at the end of the simulation and, consequently, in a larger SFR. This model is more stable against the feedback of the dying stars, so a longer duration of SF is expected. At the end of the simulation, $\sim 10^{8} M_{\odot}$ of stars are already present inside the galaxy (Fig. 2), a higher value than the estimated stellar mass of Fornax. We have to take into consideration, however, that more than $60 \%$ of these stars will die within 10 Gyr. Moreover, tidal stripping is likely to produce a substantial reduction of the stellar component (Kroupa 1997).

Model 4, with a reduced $\varepsilon_{\mathrm{SF}}$, has a higher initial gas mass (see Sect. 2) and therefore starts forming stars earlier and at a higher rate. Its attained metallicity is of the order of a few hundredth of $Z_{\odot}$, considerably lower than the observed value in Fornax. This is mostly due to how the newly produced metals are diluted in a larger fraction of pristine gas but also to the fact that the reduced injection energy rate per unit mass into the system reduces the turbulence, therefore decreasing the diffusivity of the medium and increasing the mixing timescale. This value of the global metallicity, although inconsistent with the chemical composition of Fornax, is similar to the metallicity of smaller dSphs, supporting the idea that the SF efficiency increases with the mass of the object (e.g. Lanfranchi \& Matteucci 2004). The evolution of $[\mathrm{O} / \mathrm{Fe}]$ vs. $[\mathrm{Fe} / \mathrm{H}]$ for this model shows a large loop (Fig. 3), caused by the continuous increase in total gas mass through gas inflow between $\sim 100$ and $\sim 200$ Myr that also produces a decrease in the global metallicity (see Fig. 2). Therefore, at variance with the other models, the $[\mathrm{Fe} / \mathrm{H}]$ is not a proxy of the time.

Two DM-rich models (models $1 \mathrm{dc}$ and 1de) are also considered. Their setup is identical to model 1 , but their gravity is dominated by a large static DM halo. The comparison of the SFR in these models is shown in Fig. 4. The differences between the different models are very small. Also the other properties of the models are not significantly affected by the presence of a large DM halo. This shows that the global behaviour of the gas in the central part of the galaxy is mostly determined by the total mass and distribution of gas itself rather than by the depth of the potential well. However, it is foreseeable that the late evolution of the gas is affected by the presence of a DM halo extending up to very large distances. 


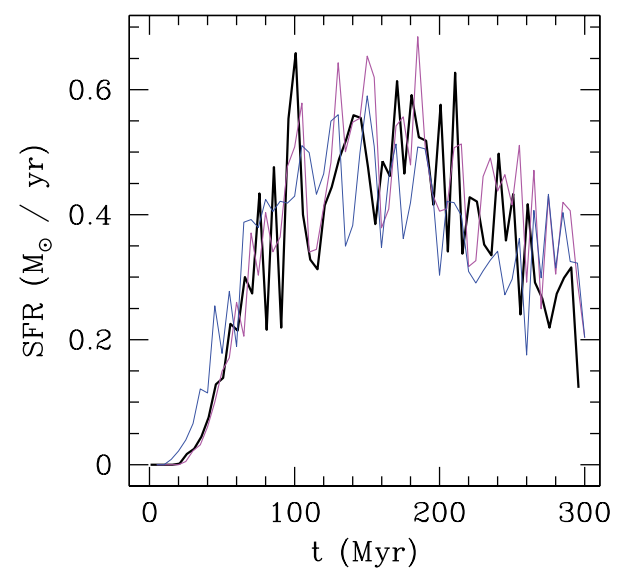

Fig. 4. Evolution with time of the SFR (in $M_{\odot} / y r$ ), for Model 1 (reference model, very thick line), Model 1de (extended DM halo, thick line), and Model 1dc (concentrated DM halo, thin line).

We also ran a simulation in which $\varepsilon_{\mathrm{SF}}=0.2$. In this case, the expanding cavity created by overlapping $\mathrm{SNe}$ is energetic enough to produce a blow-away and unbind most of the gas in the galaxy in $\sim 100 \mathrm{Myr}$. This determines the duration of the SF period, which is very short and inconsistent with the typical SF histories of Local Group dSphs (Grebel 1997).

Finally, when an asphericity in the initial distribution of gas is introduced, the gas tends to expand preferentially along one axis, namely the one in which the pressure gradient is steeper. Interestingly, the development of a polar galactic wind does not suppress the SF process, since some gas is compressed towards the walls of the galactic chimney where the condition for the onset of SF can be fulfilled (see Recchi et al. 2007).

\section{Discussion and conclusions}

By means of a detailed 2-D chemodynamical code, we have simulated the evolution of isolated dwarf spheroidal galaxies without DM in order to study the early evolution of TDGs and compared the results with DM-dominated models.

We have seen that reasonable assumptions about the SF efficiency and the temperature threshold above which the SF is suppressed lead to a SF lasting at least $400 \mathrm{Myr}$. This interval of time broadens further if we consider very small SF efficiencies (which is possible in small galaxies). Provided that it keeps its spherical symmetry, a small galaxy is therefore relatively stable against the feedback of the ongoing star formation, even in absence of a large DM halo. This issue has already been shown by Hensler et al. (2004). However, we have seen that a DM-dominated model shows a behaviour similar to the DM-free ones, indicating that the gas dynamics of the galaxy, at least in its central part, is dominated by the gas density and distribution more than by the depth of the potential well. Eventually, after several hundred Myr, a strong galactic wind may arise, able to eject most of the gas. However, this gas does not necessarily leave the parent galaxy. It may indeed later be reaccreted by the gravitational pull of the galaxy if tidal interactions do not disperse it. Recchi \& Hensler (2006) have done experiments about the timescale required to refill with cold gas the centre of a galaxy after a supernova-driven galactic wind has occurred. This timescale is of the order of a few hundred Myr, after which a new episode of SF can occur. This might lead to the bouncing back and forth of the gas, due to the competing effects of the gravitational pull and the feedback of the ongoing star formation, resulting in a sort of self-regulation, until the moment in which a close encounter with a more massive galaxy definitely moves the gas away, shutting off the cycle. This mechanism is under investigation at the present time.

A more detailed description of the code and a more extensive analysis of the parameter space will be provided in a paper in preparation. Further work and modelling is necessary to sharpen our understanding of TDGs. In particular we are including stellar dynamics and a time-dependent tidal field due to the interacting system. Also the IMF is going to be subject of detailed analysis. In particular, we will implement the integratedgalactic initial mass function (Weidner \& Kroupa 2005), in order to consider the variations of galaxy-wide IMF as a function of the SFR. Finally, we have started 3-D simulations of TDGs (Marcolini et al., in preparation).

Acknowledgements. We thank the anonymous referee whose comments improved the paper. This project is supported by the German Deutsche Forschungsgemeinschaft, $D F G$, as part of the priority programme 1177 (under grants TH 511/8 and KR1635/8-1). S.R. wishes to thank A. Rieschick for providing a subroutine for the calculation of the self-gravity and A. Marcolini for help and assistance in the development of the code.

\section{References}

Barnes, J., \& Hernquist, L. 1992, Nature, 360, 715

Bournaud, F., \& Duc, P.-A. 2006, A\&A, 456, 481

Duc, P.-A., \& Mirabel, I. F. 1998, A\&A, 333, 813

Grebel, E. K. 1997, Rev. Mex. Astron. Astroph., 10, 29

Hensler, G., Theis, C., \& Gallagher, J. S. III 2004, A\&A, 426, 25

Köppen, J., Theis, C., \& Hensler, G. 1995, A\&A, 296, 99

Kroupa, P. 1997, NewA, 2, 139

Kroupa, P. 1998, MNRAS, 300, 200

Kroupa, P., Theis, C., \& Boily, C. M. 2005, A\&A, 431, 517

Lanfranchi, G. A., \& Matteucci, F. 2004, MNRAS, 351, 1338

Leitherer, C., Schaerer, D., Goldader, J. D., et al. 1999, ApJS, 123, 3

Mastropietro, C., Moore, B., Mayer, L., Wadsley, J., \& Stadel, J. 2005, MNRAS, 363,509

Mateo, M. 1998, ARA\&A, 36, 435

Matteucci, F., \& Greggio, L. 1986, A\&A, 154, 279

Metz, M., \& Kroupa, P. 2007, MNRAS, 376, 387

Okazaki, T., \& Taniguchi, Y. 2000, ApJ, 543, 149

Recchi, S., \& Hensler, G. 2006, A\&A, 445, L39

Recchi, S., Matteucci, F., \& D'Ercole, A. 2001, MNRAS, 322, 800

Recchi, S., Matteucci, F., D’Ercole, A., \& Tosi, M. 2004, A\&A, 426, 37

Recchi, S., Kroupa, P., Theis, C., \& Hensler, G. 2007, EAS Publ. Ser., 24, 297

Rieschick, A., \& Hensler, G. 2003, Ap\&SS, 284, 861

Ružička, A., Palouš, J., \& Theis, C. 2007, A\&A, 461, 155

Tolstoy, E., Venn, K. A., Shetrone, M., et al. 2003, AJ, 125, 707

Toomre, A., \& Toomre, J. 1972, ApJ, 178, 623

Walter, F., Martin, C. L., \& Ott, J. 2006, AJ, 132, 2289

Weidner, C., \& Kroupa, P. 2005, ApJ, 625, 754

Wetzstein, M., Naab, T., \& Burkert, A. 2007, MNRAS, 375, 805 